Revue des patrimoines

46 | 2022

Le patrimoine de la Justice

\title{
Pierre Valentin Boudhors (1754-1831) : un projet de transformation de la prison à Ensisheim
}

Pierre Valentin Boudhors (1754-1831): a transformation project of the prison in Ensisheim

\section{Véronique Umbrecht}

\section{QpenEdition}

\section{Journals}

Édition électronique

URL : https://journals.openedition.org/insitu/33260

DOI : $10.4000 /$ insitu. 33260

ISSN : $1630-7305$

Éditeur

Ministère de la Culture

Référence électronique

Véronique Umbrecht, «Pierre Valentin Boudhors (1754-1831) : un projet de transformation de la prison à Ensisheim », In Situ [En ligne], 46 | 2022, mis en ligne le 18 janvier 2022, consulté le 03 février 2022. URL : http://journals.openedition.org/insitu/33260 ; DOI : https://doi.org/10.4000/insitu.33260

Ce document a été généré automatiquement le 3 février 2022

\section{(i)}

In Situ Revues des patrimoines est mis à disposition selon les termes de la licence Creative Commons Attribution - Pas d'Utilisation Commerciale - Pas de Modification 4.0 International. 
Pierre Valentin Boudhors (1754-1831) : un projet de transformation de la prison à Ensisheim Pierre Valentin Boudhors (1754-1831): a transformation project of the prison in
Ensisheim

Véronique Umbrecht

1 Le projet de transformation du dépôt de mendicité d'Ensisheim (Haut-Rhin) de 1806 survient dans un contexte de transformation du système pénitentiaire français. Depuis le siècle précédent, de nombreux intellectuels avaient dénoncé les conditions inhumaines de détention des prisonniers. Une réflexion judiciaire, éthique, administrative introduit un nouveau modèle architectural de prison qui participe de l'éducation du citoyen. Ce schéma doit afficher son efficacité éthique : la prison n'a de justification que dans la réinsertion du condamné dans la société. Sollicité par le préfet du Haut-Rhin afin de remanier l'ancien dépôt de mendicité, l'architecte de la Ville de Strasbourg, Pierre-Valentin Boudhors, réalise un projet ambitieux s'appuyant sur son Discours préliminaire pour expliciter son programme. Formé à Paris notamment dans le cabinet de Chalgrin, il suit le sillon tracé par les architectes néo-classiques pour qui l'architecture était un acte moral. À Ensisheim, Boudhors développe une « architecture parlante " et y mêle les éléments architecturaux antiquisants, médiévaux et de la Renaissance italienne. Mais l'ensemble de ses dessins puise l'essentiel de son inspiration dans la saline d'Arc-et-Senans de Claude Nicolas Ledoux. 


\section{Pierre-Valentin Boudhors, un architecte singulier}

2 Lorsque le préfet du Haut-Rhin Félix Desportes sollicite Pierre Valentin Boudhors en 1806, l'architecte en chef de Strasbourg avait entamé une seconde carrière au service de sa ville natale depuis peu.

Il naît en 1754 à Strasbourg. Fils d'un inspecteur des Ponts et Chaussées, il commence sa formation à l'école municipale des arts et métiers. Cette institution dispensait des cours de dessin gratuits et préparait à l'apprentissage d'un métier en initiant les élèves au dessin technique. Il obtient son diplôme en 1772. Son apprentissage aurait pu se poursuivre sur les chantiers en suivant son père et ses collègues. C'était sans compter avec l'ambition du préteur royal François Baron d'Autigny. En 1773, la santé de l'architecte en chef de Strasbourg Samuel Werner décline rapidement. La question de sa succession se pose. Grâce certainement à la situation de son père et à la protection du préteur royal, Boudhors devient l'adjoint de Werner en 1774, âgé d'à peine vingt ans. Sous la pression de «l'homme du roi », les édiles municipaux décident de financer les études à Paris du jeune inspecteur des Bâtiments ${ }^{1}$. Boudhors commence alors son cursus parisien au Collège des Quatre Nations, puis suit les cours de Jacques François Blondel à l'Académie royale d'architecture. Recommandé par son condisciple et ami Jean-Baptiste Kléber, parti poursuivre sa formation dans le cabinet de Jean-François Thérèse Chalgrin, Boudhors entre au service du célèbre architecte parisien.

Lors de son séjour à Paris, Boudhors bénéficie d'un environnement culturel de premier plan. À l'Académie royale d'architecture, il acquiert les principes du classicisme blondélien. Dans l'agence de Chalgrin, il bénéficie des enseignements de l'un des chantres du nouveau style. Formé par Servandoni et Etienne Louis Boullée, Grand Prix de Rome en 1758, Chalgrin avait tiré les leçons des monuments antiques lors son voyage d'étude dans la «Ville éternelle ». De retour à Paris, il fait partie de la jeune génération d'architectes qui s'opposent à leurs maîtres classicisants et qui cherchent de nouvelles voies dans l'étude du patrimoine passé. Ces artistes aspiraient à une conception plus épurée de l'art de construire faisant référence aux monuments antiques et aux œuvres de la Renaissance. Premier architecte et intendant des Bâtiment de Monsieur, le comte de Provence, Chalgrin s'était constitué une vaste clientèle dans l'aristocratie et la haute bourgeoisie. Le jeune Boudhors profite de la notoriété de son maître et participe notamment aux travaux du Palais Bourbon commandé par Monsieur. En 1775, il est chargé de coordonner le chantier du Mausolée du Maréchal de Saxe à l'église SaintThomas de Strasbourg, sculpté par Jean Baptiste Pigalle. C'est l'occasion pour l'apprenti-architecte de se faire valoir auprès de son patron.

Rentré à Strasbourg en 1777 et fort de ces années de formation parisiennes, Boudhors tente d'introduire le néo-classicisme à Strasbourg. Il eut également l'occasion à plusieurs reprises de séjourner à Paris et de voir les réalisations de ses illustres confrères tels Claude Nicolas Ledoux ou Marie Joseph Peyre et Charles de Wailly. Primitivement encouragé par son protecteur, il multiplie les projets de bâtiments publics qui sont sans doute mal compris par des édiles locaux, peu réceptifs aux nouveautés venues de la capitale. Il perd peu à peu le soutien des membres du Magistrat alors que le préteur royal Baron d'Autigny avait été appelé à d'autres destinés. En 1789, il est limogé. Les années de tourmente révolutionnaires passées, Boudhors est réintégré dans ses fonctions d'architecte municipal au début des années 1800. Il est alors chargé de revoir les plans du nouveau théâtre, après l'incendie de 
l'ancienne salle de spectacle 2 . C'est à cette même période que Boudhors est contacté par le préfet du Haut-Rhin Félix Desportes afin de dessiner les plans de rénovation de la prison d'Ensisheim.

\section{Le projet de prison d'Ensisheim dans le contexte de prison de la fin du XVIII e siècle et de l'Empire}

6 La transformation du dépôt de mendicité en prison centrale à Ensisheim s'inscrivait dans une démarche hygiéniste qui débuta à la fin du XVIII ${ }^{e}$ siècle.

\section{La question de l'enfermement}

7 Depuis plusieurs siècles, le pouvoir central avait la volonté de contrôler la population itinérante de mendiants et de vagabonds qui encombrait les rues des villes. Une politique de l'enfermement avait primitivement été mise en place mais au XVII ${ }^{\mathrm{e}}$ siècle, une refonte du système hospitalier devait voir le jour, accueillant ces nécessiteux. Faute de moyens financiers et matériels, les hôpitaux furent contraints de les renvoyer. On revint au principe de l'enfermement sans régler le problème de l'indigence. La Déclaration de 1764 relative à la «vagabonnerie» condamnait aux galères les vagabonds valides et à la prison les impotents, les vieillards et les femmes seules. Ce texte était confirmé par l'arrêt du Conseil d'État du 21 octobre 1767 qui marquait la création des dépôts de mendicité. Bien que ces lieux fussent de fait des prisons, la volonté du pouvoir central était de mettre au travail les détenus valides. Une organisation administrative se mit en place sous la responsabilité de l'État en la personne de l'intendant. La mission de ces dépôts ne consistait pas uniquement à retirer de la circulation des gueux de toutes sortes mais ces prisons avaient développé au fil des décennies une fonction hospitalière ${ }^{3}$.

8 Cependant, la situation des prisonniers et l'état désastreux des dépôts de mendicité et des nombreuses prisons ordinaires du royaume soulevaient beaucoup d'interrogations. Les intellectuels s'emparèrent de ce sujet de l'incarcération. L'ouvrage de l'italien Cesare Beccaria, traduit par l'abbé Morellet, suscita l'enthousiasme des philosophes français. Son Traité des délits et des peines, paru en 1764, dressait un tableau synthétique de l'état des prisons et de la justice criminelle tout en tentant d'en tirer des enseignements. Quelques années plus tard, l'anglais John Howard publia en 1777 The State of the Prisons qui faisait le même constat dans les prisons britanniques. En France, Louis XVI avait fortement limité les lettres de cachet et supprimé la Question lors des interrogatoires. Le ministre Necker avait demandé à l'Académie royale des sciences d'établir un état des lieux des prisons. En 1784, Antoine Laurent de Lavoisier rendit le Rapport sur les prisons qui préconisait notamment l'éloignement des prisons des centres urbains pour des raisons d'hygiène et de sécurité de la population ${ }^{4}$. En 1780, Malesherbes fit transformer par l'architecte Boullée un ancien hôtel particulier en prison modèle : la prison de La Force. Les détenus étaient séparés par sexe et par type de peine et leurs conditions de vie étaient améliorées. Les tentatives ministérielles de réorganisation du système pénitentiaire avaient mises en avant la question du travail des détenus. Il s'agissait non seulement de lutter contre l'oisiveté mais également de soulager le budget de l'État avec le fruit de leur travail. 
9 La réflexion se poursuivait dans le domaine législatif mais les avancées en terme judiciaire restèrent minimes. La Révolution relança le débat avec l'augmentation substantielle des incarcérations; la circulaire ministérielle du 5 février 1796 intimait l'ordre aux autorités locales de mettre en place de nouvelles prisons telles que prévues par les textes. Faute d'argent, les bâtiments conventuels réquisitionnés comme biens nationaux furent transformés à la hâte en maisons d'arrêt, de justice ou de correction.

10 Un tournant s'est opéré dans l'histoire des prisons avec Napoléon Bonaparte. C'est une refonte administrative et judiciaire qui se profile au début du XIX ${ }^{\mathrm{e}}$ siècle. Les premières maisons centrales sont installées en 1800 dans les pays fraîchement conquis comme en Belgique, à Gand ou Vilvoorde. Sur le territoire français, un maillage de prisons est implanté au centre de circonscriptions regroupant plusieurs départements, le long des frontières ou dans des zones militairement stratégiques. Ces nouveaux établissements pénitentiaires deviennent alors les instruments de lutte contre la désertion et l'insoumission. Les programmes de construction sont lancés sous le contrôle financier et administratif des préfets et transcrivent la volonté politique de mettre au travail les détenus. En effet, ces maisons centrales sont organisées comme de grandes manufactures textiles où les hommes tissent, les femmes filent. Cette main d'œuvre bon marché a participé à l'émergence de dynastie d'industriels entrepreneurs tels les Dollfuss à Mulhouse 5 .

11 Cette ligne conductrice se confirme dans les premières décennies du XIX ${ }^{\mathrm{e}}$ siècle.

\section{Le programme architectural de la prison}

12 Les changements législatifs et doctrinaires ne tardent pas à se traduire dans l'architecture. Le thème de la prison est omniprésent au XIX ${ }^{\mathrm{e}}$ siècle. Les prémices apparaissent au siècle précédent puisque l'Académie royale d'architecture soumet au concours de 1768 et de 1778 le sujet de la prison publique dont l'argumentaire était d'imaginer une prison sur un terrain isolé de grande dimension et hors des centres urbains. Ce nouveau sujet n'est pas éloigné des réflexions urbanistiques qui émergent à cette époque sur les miasmes urbains, la circulation de l'air et de l'eau, sur les méfaits physiques et moraux de l'entassement des populations des villes ${ }^{6}$. Jacques François Blondel écrit dans son Cours d'architecture : «[...] en général elles [les prisons de Paris] ne sont ni assez grandes ni assez aérées : nous pensons d'ailleurs qu'il est contre toute vraisemblance de confondre, dans une même enceinte, les citoyens détenus pour dettes avec des criminels qui, à toute sorte d'égards, méritent les fers ${ }^{7}$." Les architectes s'emparent de cette question et imaginent les prisons comme de véritables «théâtres punitifs » à l'instar de la prison de Newgate à Londres construite par Dance le jeune entre 1764 et 1784 . La façade extérieure ressemble à un château fort percé seulement de quelques baies, sensée effrayer le spectateur. Pierre Desmaisons dote La Petite Force d'une façade spectaculaire sur les dessins de Boullée. L'ouvrage qui influence le plus les architectes du début du XIX ${ }^{\mathrm{e}}$ siècle est le Panoptique de Jérémy Bentham ${ }^{8}$. Traduit et publié en France en 1791, l'ouvrage présente une réflexion novatrice sur le mode de détention des prisonniers. Bruno Foucart définit ce projet ainsi : «[...] trouver un système qui allège le poids des murs et des verrous en permettant de surveiller une communauté de la manière la plus simple et la moins contraignante ${ }^{9}$. » Chaque détenu est isolé dans une cellule et n'est autorisé à se déplacer que pour se rendre dans les ateliers ou pour se promener une fois par jour. Bentham invente un édifice circulaire 
composé des cellules des prisonniers dont le centre est occupé par une grande tour où est installé le bureau des surveillants. La vue périphérique du bâtiment depuis la tour centrale nécessite peu de personnel, mais l'implantation du bâtiment demande une superficie de terrain importante. Bien que difficile à mettre en place et fort coûteux, le Panoptique de Bentham a marqué l'imaginaire des penseurs du pénitentiaire: le système cellulaire est un principe architectural qui fait florès en France au cours du XIX ${ }^{\mathrm{e}}$ siècle.

\section{Une architecture carcérale parlante à Ensisheim}

13 L'Alsace est une région frontalière, l'administration pénitentiaire impériale avait la volonté de la doter de prisons centrales. Sous la Révolution, Strasbourg, capitale de l'ancienne province, était pourvue de cinq prisons ordinaires et militaires. Selon son importance administrative, chaque ville était en capacité de détenir des prisonniers dans des conditions souvent très précaires ${ }^{10}$.

En 1806, le ministre de l'Intérieur charge le préfet du Haut-Rhin Félix Desportes de transformer le dépôt de mendicité d'Ensisheim (Haut-Rhin) en maison centrale de détention. Cette nouvelle prison devait étendre sa juridiction sur dix départements.

Les premiers bâtiments du dépôt de mendicité remontent au début du XVII ${ }^{\mathrm{e}}$ siècle. Lors du rattachement de l'Alsace au royaume de France au traité de Westphalie, les Jésuites français s'installent dans l'ancien hospice médiéval transformé en collège au $\mathrm{XVI}^{\mathrm{e}}$ siècle. Ils rénovent les différents bâtiments et construisent une église baroque. Lorsque les Jésuites sont chassés de France en 1765, le site est abandonné quelques années avant d'être transformé en dépôt de mendicité en $1776^{11}$.

Une architecture vue sous l'angle de la salubrité et de la sûreté

Lorsque Pierre Valentin Boudhors se transporte à Ensisheim en 1806, il fait des relevés des bâtiments existants et constate dans son Discours préliminaire sur l'établissement en général des prisons que "[...] les bâtiments actuels de la maison d'Ensisheim quoique considérables ne présentent point les moyens d'en faire une maison centrale de détention aussi étendue que l'on avait pu le supposer, et s'il fallait se tenir au local actuel, il faudrait renoncer à l'idée de pouvoir comparer cet établissement avec ceux cités par la lettre susdite de S. E. le ministre. Cet établissement ne contenait autrefois que 150 à 200 prisonniers et il faudrait déjà bien entamer le service si le nombre devait être porté au double dans le local actuel, les dégradations du bâtiment exigent d'ailleurs des réparations si considérables que la dépense à faire ne serait point suffisamment balancé par l'utilité que l'on en tirerait si l'on voulait se contenter dans ce qui existe ${ }^{12} \ldots$ ». Il envisage alors de partir de l'existant et d'étendre son projet. Un plan masse donne une vision d'ensemble de la transformation de l'ancien dépôt de mendicité [fig. $\left.{ }^{\circ} 1\right]$. 
Figure 1

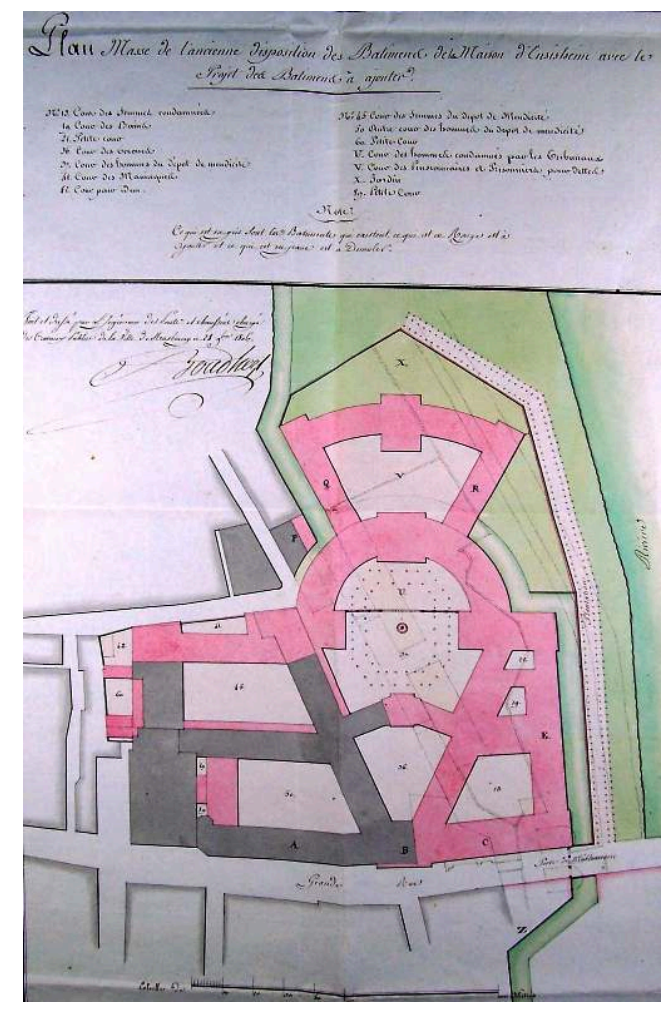

Plan masse de l'ancienne disposition des bâtiments de la maison à Ensisheim avec le projet des bâtiments à ajouter : $n^{\circ} 13$ cour des femmes condamnées, $n^{\circ} 19$ cour des bains, $n^{\circ} 21$ petite cour, $n^{\circ} 37$ cour des hommes du dépôt de mendicité, $n^{\circ} 41$ cour des maniaques, $n^{\circ} 42$ cour pour idem, $n^{\circ} 45$ cour des femmes du dépôt de mendicité, $n^{\circ} 50$ autre cour des hommes du dépôt de mendicité, $n^{\circ} 60$ petite cour, $n^{\circ} 89$ petite cour, $\mathrm{U}$ cour des hommes condamnés par les tribunaux, $\mathrm{V}$ cour des pensionnaires et prisonniers pour dettes, $\mathrm{X}$ jardin, Pierre Valentin Boudhors, 1806, conservé aux archives départementales du Haut-Rhin (Y 218).

Reproduction Véronique Umbrecht.

Suivant les préceptes des théoriciens du judiciaire, les différents corps de bâtiments sont répartis selon le type de détenus: "Nous avons partagé le tout en cinq parties inégales, les deux plus grands quartiers sont pour le dépôt général de mendicité et pour les condamnés par les tribunaux, la $3^{\mathrm{e}}$ partie pour les prisonniers pour dettes et ceux pour lesquels on paye pensions, la $4^{\mathrm{e}}$ pour des prisonniers d'État et la $5^{\mathrm{e}}$ pour les maniaques que nous croyons devoir se trouver plutôt avec le dépôt des pauvres que dans les hôpitaux ordinaires. » Boudhors choisit de placer celui-ci dans l'ancien édifice et les condamnés par les tribunaux dans les nouveaux locaux. Fidèle à ses maîtres parisiens, Boudhors élabore un ensemble régulier: "J'avais cependant exigé de concentrer mon projet dans l'ancien local ; [...] j'y ai totalement renoncé en prenant le parti d'utiliser tous les bâtiments élevés à plusieurs étages pour ne démolir que de mauvaises constructions le long du canal [...], en faisant une disposition nouvelle qui puisse former un ensemble régulier avec ce qui existe de bon de l'ancien établissement et tout en régularisant la disposition des corps de bâtiments et en les séparant par des cours qui se scindent dans un ordre symétrique. »Cette disposition s'inspire des rares projets de prison réalisés par des architectes du XVIII siècle. Claude Nicolas Ledoux avait dessiné un ensemble architectural en 1777 composé d'un palais de justice et d'une prison à Aix-en-Provence. Le plan de la maison d'arrêt présentait la forme d'une croix grecque flanquée de quatre cours carrées plantées d'arbres. Un système de circulation 
fait de galeries et de promenoirs devait entouré chaque espace. À Ensisheim, ce schéma est adapté au terrain et aux bâtiments existants. L'architecte jongle avec les formes géométriques, chères au néo-classicisme ; il dessine un long rectangle auquel il insère un demi-cercle, lui-même terminé par un trapèze. À l'intérieur, il établit une symétrie dans l'axe du portail d'entrée. Ce jeu de figures lui permet de placer différentes cours assez vastes pour y recevoir les détenus.

L'hygiène et la salubrité transparaissent dans l'agencement des corps de bâtiments. Ils avaient été l'objet de réflexions des intellectuels depuis plusieurs décennies. La promiscuité, l'humidité et le manque d'eau potable avaient été une des causes de la surmortalité carcérale. Boudhors élabore un projet qui reprend le système cellulaire c'est-à-dire un détenu par chambre; il trace des larges cours bien aérées dont la plus importante est plantée d'arbres et possède une fontaine. Des jardins entre le mur d'enceinte et les bâtiments sont prévus afin d'apporter un peu d'humanité. Il décrit son dispositif d'adduction d'eau : «[...] J'ai détourné le canal depuis le vis-à-vis des écuries de la gendarmerie $\mathbf{P}$ pour le faire passer sous les bâtiments $\mathbf{Q}$ et $\mathbf{R}$ et une voûte sous la cour $\mathbf{V}$ pour que ses eaux puissent laver à leur passage les latrines des nouveaux corps de bâtiment $\mathbf{E}$ pour se jeter dans son ancien lit au point $\mathbf{Z}$ [...]. » et «[...] en place de la grande partie des canaux actuellement répandu dans l'ancien bâtiment et qui rendent plutôt la maison humide et malsaine que salubre; nous avons conservé que ceux nécessaires pour l'écoulement des eaux et des latrines et que pour rafraichir l'air des cours, nous proposons de faire couler dans la saison nécessaire dans de certaines heures du jour une eau dans les rigoles des pavés des cours au moyen de leur machine hydraulique qui élèvera l'eau du canal cette méthode est infiniment préférable à cette quantité d'aqueducs sous terrain qui répandent une humidité désastreuse pour les murs et les habitants de la dite maison». Les plans signalent des bains ( $\left.n^{\circ} 19\right)$ qui semblent bien petits au regard du nombre de prisonniers prévu. Néanmoins, la notion de l'hygiène a été prise en compte par l'architecte.

La sûreté est un autre domaine qui relève d'une prison. Selon la dangerosité des détenus, les cellules sont soit placées au centre de l'édifice comme celles des hommes condamnés par les tribunaux (partie en demi-cercle, $\mathbf{U}$ ), soit donnant sur les murs extérieurs comme celles des détenus pour dettes (partie en trapèze, V). L'interaction entre les bâtiments ne peut se faire à la légère. Bien qu'exprimé dans un contexte industriel, Daniel Rabreau souligne que « la logique du système productif, fondé sur le rendement, la surveillance et la sécurité, recommande la combinaison de la courbe et de ses segments et rayons; la saline sera donc un panoptique ${ }^{13} . .$. ». Ledoux avait donc détourné l'objectif premier du modèle de Bentham mais le principe d'un contrôle efficient prévalait également à Arc-et-Senans. Lors de ses études à Paris et de ses voyages dans la capitale après 1777 , Boudhors a certainement vu les réalisations de Ledoux, comme en témoignent ses divers projets pour Strasbourg ${ }^{14}$. Une visite à la saline royale, proche de l'Alsace, a dû également faire partie de son cursus de formation. Quant au modèle du Panoptique, il ne pouvait pas être repris dans son ensemble puisque le terrain et le bâti existant ne le permettaient pas mais Boudhors l'adapte en gardant l'idée de bâtiments semi-circulaires ou en segment d'arc, ponctués de hautes tours de surveillance. Un dispositif dense de galeries et de couloirs qui communiquent d'un bâtiment à l'autre court le long des différentes cellules [fig. ${ }^{\circ} 2$ et fig. $\left.{ }^{\circ}\right]$. 
Figure 2

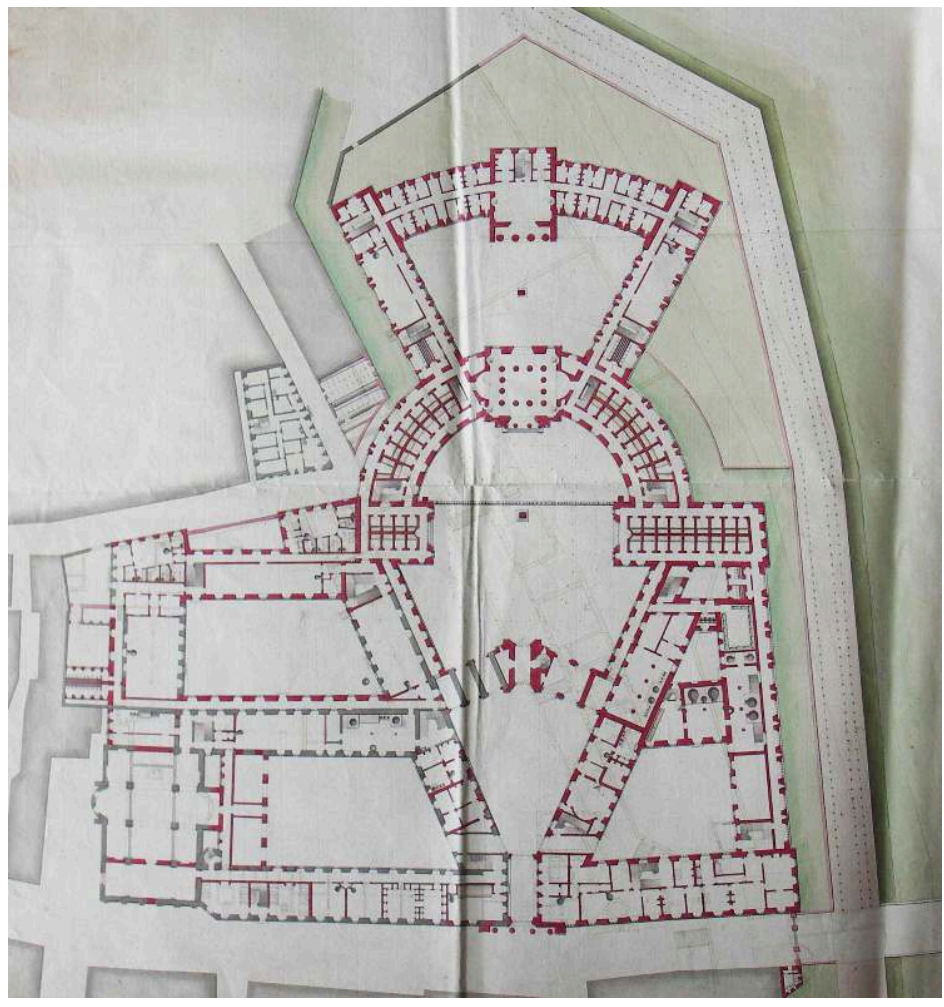

Projet de maison centrale à Ensisheim : plan du rez-de-chaussée, Pierre Valentin Boudhors, conservé aux archives de la Ville et de l'Eurométropole de Strasbourg (315 WM 11).

Reproduction service photographique des archives de la Ville et de l'Eurométropole de Strasbourg (AVES). 
Figure 3

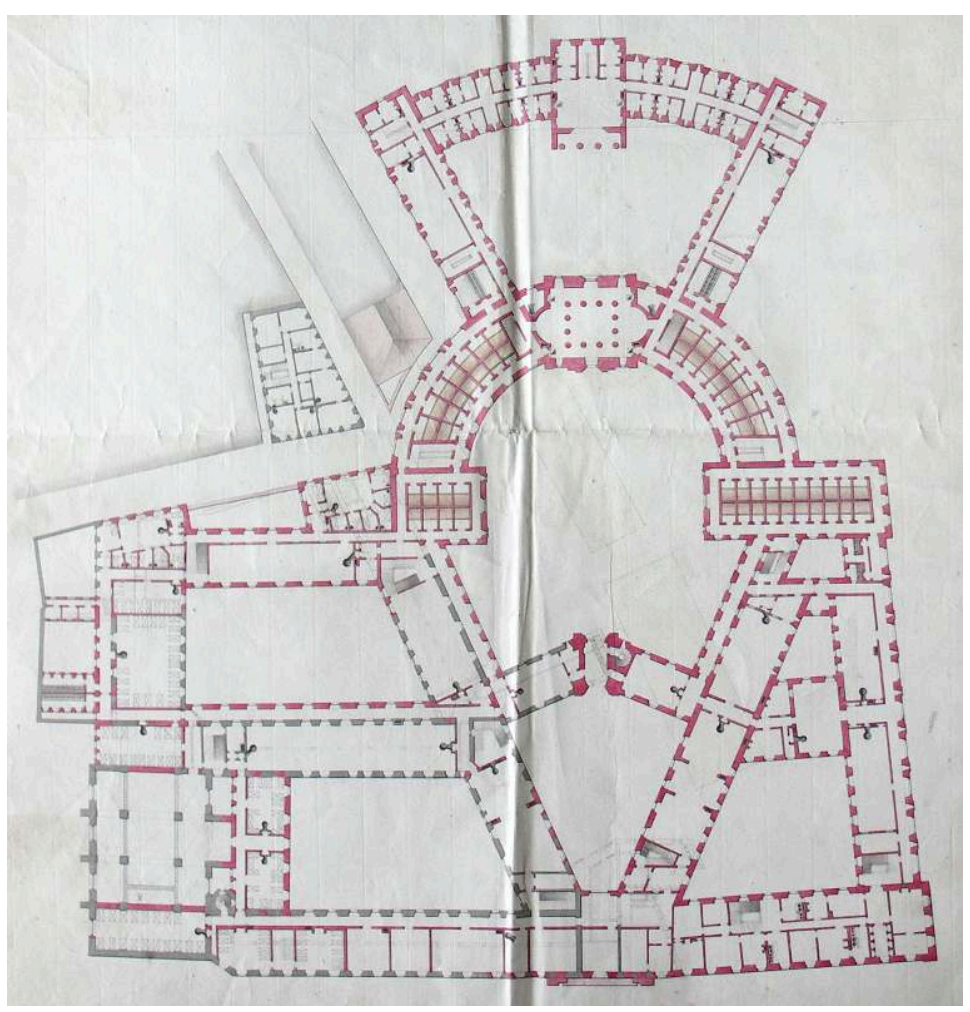

Projet de maison centrale à Ensisheim : plan du premier étage, Pierre Valentin Boudhors, conservé aux archives de la Ville et de l'Eurométropole de Strasbourg (315 WM 12)

Reproduction service photographique des archives de la Ville et de l'Eurométropole de Strasbourg (AVES).

21 Un poste de gendarmerie $\mathbf{P}$ qui se trouve à l'extérieur mais tout de même proche de la prison vient renforcer le maintien de l'ordre. La sécurité des détenus passe également par la séparation des sexes. Dans le dépôt de mendicité les femmes ont leur propre cour tout comme celles condamnées par les tribunaux. La protection de ces «locataires" particuliers est primordiale puisque la prison a une vocation éducative: elle doit donner « les moyens de faire du prisonnier un homme qui puisse un jour rentrer dans la société ${ }^{15}$ ». Cette rédemption s'obtient par le travail qui empêche tout amollissement des capacités physiques et morales des individus enfermés. Quelles activités sont proposées à la maison centrale? Aucun document d'archive, ni le Discours préliminaire ne le précisent. Comme Ensisheim était proche du bassin de l'industrie textile, le travail devait certainement consister à l'épluchement du coton ${ }^{16}$. À l'analyse des plans de la prison, il semble que les ateliers se soient situés au rez-de-chaussée; divers bacs sont mentionnés aussi bien dans le dépôt que dans la partie réservée aux condamnés.

La distribution générale des différents corps de bâtiments révèle la volonté humaniste de l'architecte. Par son caractère fonctionnel et hygiéniste, le projet de maison centrale affirme son appartenance à une architecture parlante novatrice en Alsace au début du XIX siècle. 


\section{Le programme du décor monumental}

Le caractère de la prison participe de l'éducation du détenu. Par ses formes, celle-ci doit exprimer l'enfermement et la punition. Les architectes utopistes tels que Ledoux, Boullée ou Lequeu l'avaient pensée comme un monument public dont l'architecture traduisait une justice exemplaire. Dans son Discours préliminaire, Boudhors était bien conscient de réaliser un édifice à haute valeur morale. Il débute son préambule ainsi : «Les facultés que les hommes ont reçu de la nature les destinent à des actions grandes et généreuses; mais hélas! Il en est beaucoup parmi eux qui en abusent, et qui les emploient pour nuire à la société. [...] La manière de construire ces bâtiments a mérité toute l'attention du gouvernement, c'est d'elle que dépend en grande partie la sûreté publique ; elle intéresse encore l'humanité. » Même si la construction de prison relève de l'État, l'architecte qui a charge de la dessiner fait œuvre sociale. Comment Boudhors a-t-il traduit ces idéaux dans la pierre? Malheureusement, les archives ne conservent que trois élévations du projet d'Ensisheim ${ }^{17}$. Ces dessins représentent les corps de bâtiments qui séparent les cours placées dans l'axe de l'entrée principale. Le décor architectural est concentré sur les ressauts centraux tandis que l'ensemble des façades tranche par son austérité. Le vocabulaire utilisé par Boudhors puise son inspiration dans l'histoire de l'art antique, de la Renaissance et du classicisme mais il le réinterprète sous le prisme de Piranèse. Les vedute de prisons de l'architecte-graveur vénitien, publiées entre 1745 et 1760 avaient été largement diffusées dans toute l'Europe et avaient marqué la génération des architectes de la seconde moitié du $\mathrm{XVIII}^{\mathrm{e}}$ siècle $^{18}$. Ces gravures, libres de toute vérité archéologique, exprimaient l'imaginaire du dessinateur au service d'une architecture parlante.

Après avoir traversé le portail d'entrée de la prison d'Ensisheim, le détenu pénètre dans la première cour [fig. 4]. 


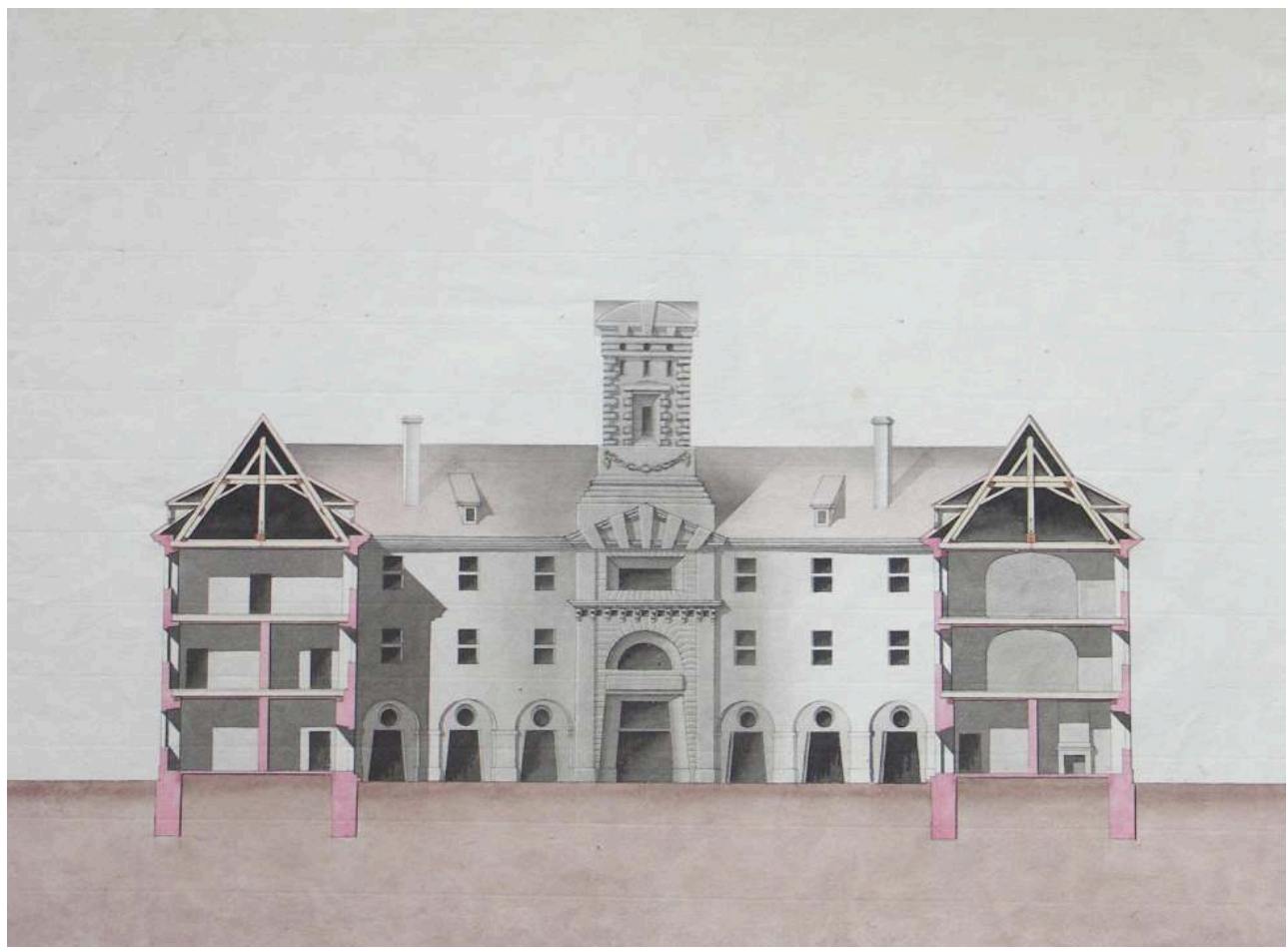

Projet de maison centrale à Ensisheim : élévation de la première cour, Pierre Valentin Boudhors, conservé aux archives de la Ville et de l'Eurométropole de Strasbourg (315 MW 7)

Reproduction service photographique des archives de la Ville et de l'Eurométropole de Strasbourg (AVES).

D'emblée, il se trouve face à une haute façade qui allie le fonctionnel et le symbolique. En effet, ce corps de bâtiment est traité dans son soubassement comme un vaste passage couvert. Boudhors met en scène ici une allégorie du monde chtonien. Une série de six portes empruntent la modénature des hypogées étrusques. Dans les linteaux, un oculus tel un œil divin surveille les prisonniers dans la cour. Le ressaut central est percé d'une porte colossale qui reprend le modèle de ses voisines. Il est surmonté d'un tympan à claveaux en saillie qui rappelle l'appareillage à bossage du pavillon de Philibert de l'Orme ${ }^{19}$. Juchée sur le toit, la tour de surveillance est marquée de chaînages harpés et percée de nombreuses ouvertures aux formes variées qui s'inspire également de cette architecture de la Renaissance française. Afin de rappeler au détenu et au visiteur la fonction de l'édifice, le motif de la guirlande rocaille est transformée en chaîne rompue. Une impression d'austérité et de monumentalité se dégage de cette façade qui s'inspire de l'architecture castrale maniériste.

L'ambiance change avec la façade de la deuxième cour [fig. ${ }^{\circ}$ ] . Le ressaut central présente des dimensions plus imposantes. Le rez-de-chaussée présente un portique qui cache une grotte tandis que les deux niveaux supérieurs reprennent les motifs de la première façade. Le portique associé à la grotte fait certainement référence à la porte d'entrée d'Arc-et-Senans. 


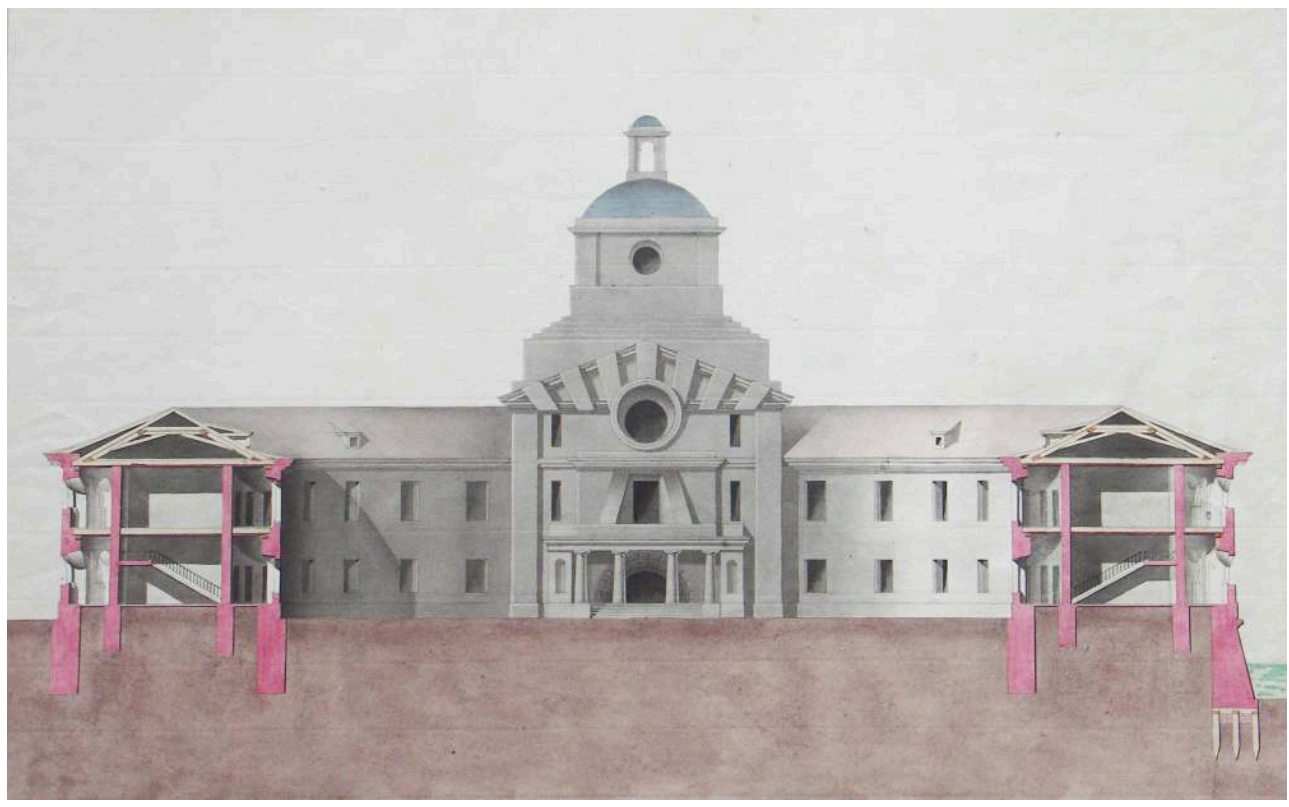

Projet de maison centrale à Ensisheim : élévation de la deuxième cour, Pierre Valentin Boudhors, conservé aux archives de la Ville et de l'Eurométropole de Strasbourg (AVES, 315 MW 6).

Reproduction service photographique des archives de la Ville et de l'Eurométropole de Strasbourg (AVES).

Ce modèle architectural renvoie à l'esthétique des jardins du XVIII ${ }^{e}$ siècle qui l'assimile au temple de la nature. Les architectes utopistes s'étaient emparés de cette forme de la grotte-temple qui puise son inspiration dans la Renaissance et le maniérisme ${ }^{20}$. Monique Mosser compare la grotte de la saline royale à une caverne, symbole de la terre, lieu de réflexion par excellence, « ... mais il apparaît vite que cette forme créée par la nature ne constitue pas une fin en soi. Elle n'est qu'un passage, une antichambre qui renvoie aux colonnes de l'entrée, c'est-à-dire, par-delà la transparente allusion au temple, à la lumière donc à la connaissance. Ainsi l'entrée monumentale d'Arc-etSenans correspond bien au lieu initiatique par excellence, mais il ne faut pas oublier qu'il s'agit en même temps d'une porte qui donne accès au "saint des saints" de la ville utopique, à la Saline elle-même ${ }^{21}$ ». Cette grotte peut paraître incongrue dans le contexte carcéral. À l'analyse des plans du rez-de-chaussée et du premier étage, il s'avère que cette entrée débouche sur un large espace à l'italienne, jalonné de colonnes formant rectangle et de deux espaces formant absides. Or, les prisons avaient également une vocation salvatrice; une chapelle y avait toute sa place. À Ensisheim, le dépôt de mendicité utilisait l'ancienne église baroque des Jésuites mais le projet de Boudhors nécessitait de la réduire de moitié. L'architecte pensait-il que deux lieux de culte seraient utiles pour une population carcérale aussi importante? Un autre indice permet d'étayer cette hypothèse. La modénature du ressaut central présente une façade de temple antique avec un tympan à claveaux harpés. Le tout est surmonté d'une tour terminée par un lanterneau qui ressemble plus à un clocher qu'à une tour de guet. Peut-être un rappel de l'ancienne église jésuite du dépôt.

La dernière élévation conservée aux archives devait se situer dans la troisième cour [fig. ${ }^{\circ}$ ]. 


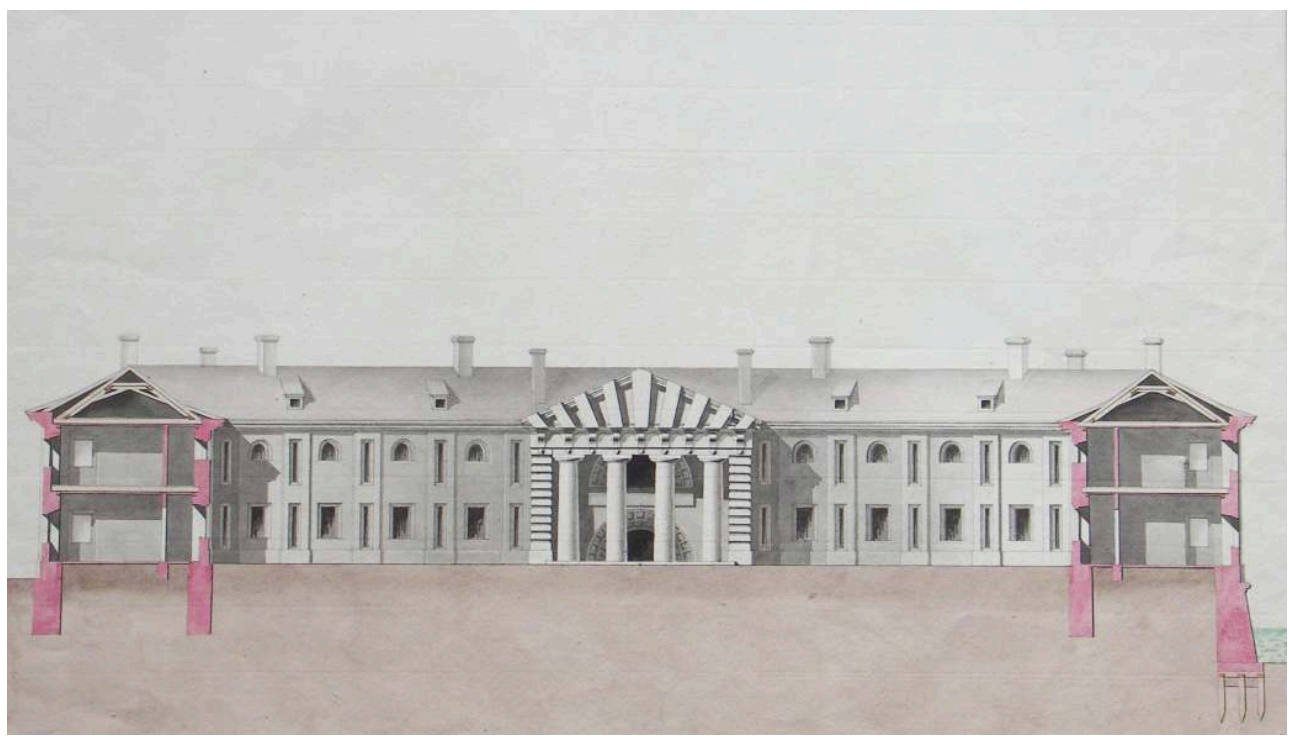

Projet de maison centrale à Ensisheim : élévation de la troisième cour, conservé aux archives de la Ville et de l'Eurométropole de Strasbourg (315 MW 8)

Reproduction service photographique des archives de la Ville et de l'Eurométropole de Strasbourg (AVES).

L'inspiration ledolcienne est plus prégnante. D'emblée, la référence à l'entrée d'Arc-etSenans saute aux yeux avec son portique dorique qui abrite deux grottes et surmonté d'un tympan à claveaux harpés. L'association de ces éléments architecturaux emprunte aux formes élémentaires issues de la nature ${ }^{22}$. L'utilisation du dorique grec dans le portique renvoie à l'art antique de Paestum, ordre primitif sans base, à fût lisse et tronconique et chapiteau composé uniquement d'une échine et d'un tailloir. Selon Monique Mosser: «Il semble bien que le "dorique de Pestum" soit, [...], une sorte d'ordre pur, d'ordre d'avant les ordres, en un mot, d'ordre poétique qui permette de conférer aux architecture un supplément d'expressivité. C'est l'ordre des prisons et des cryptes, donc du domaine souterrain ${ }^{23}$. " Le portique est surmonté d'un entablement lisse où les triglyphes sont remplacés par des consoles baroques venant soutenir les claveaux du tympan. Boudhors utilise différentes éléments architecturaux de l'histoire de la stéréotomie et les replace à sa guise dans un jeu de contrastes entre les creux et les saillies. Derrière la colonnade se cachent deux grottes superposées. Contrairement à celle de la saline royale, la caverne n'est pas taillée en pierres dégrossies mais elle est traitée comme une voûte en cul-de-four à caissons. La référence serait également à chercher dans les œuvres parisiennes de Ledoux. Le portail d'entrée de l'hôtel Guimard, la façade de l'hôtel d'Évry ou la barrière de la Conférence ont été dotés de telles voûtes. La grotte, élément du langage de l'art des jardins, est réinterprétée tout en gardant sa charge expressive et pédagogique: le détenu pénètre dans un monde souterrain propice à la rédemption. À l'instar de Ledoux, Boudhors est un adepte des effets piranésiens inscrits dans la pierre avec ses bossages vigoureux, ses contrastes abrupts de masse et d'échelle.

Le projet de prison fut refusé par le ministre de l'Intérieur qui, dans une lettre au préfet du Haut-Rhin, critiquait l'ampleur, le coût et l'esthétique du projet : « [...] ces plans ont grand besoin d'être rectifiés, et dégagés surtout de cette complication de toutes sortes de formes demi rondes trapézoïdales, barlongues à angles rentrants et saillants, obtus, 
aigus; il semblerait que l'architecte s'est attaché à employer toutes les figures géométriques et qu'il en a écarté les plus simples comme celles à angles droits ou parfaitement circulaires. En effet l'aspect de ce plan offre plutôt la figure d'une fortification que celle d'un bâtiment à usages civils ${ }^{24}$. » et il demande que l'architecte revoit sa copie. La critique est sévère pour un projet qui avait la volonté d'allier les aspects fonctionnels à une architecture empreinte d'une symbolique forte.

Dans un courrier au préfet Desportes daté du 7 août 1807, Boudhors explique qu'il lui sera impossible de revoir le projet par manque de temps: «Je ne demande aucune indemnité pour le premier projet; il me servira utilement dans mon recueil de Bâtiments publics de tout genre ${ }^{25}$." Marchait-il sur les traces de son illustre prédécesseur Ledoux qui avait publié son ouvrage en 1804 ? Il est un fait que nombre d'architectes avaient voulu laisser une trace de leur œuvre. Ce recueil est resté dans l'esprit de son auteur mais il aurait certainement montré sa puissance créatrice. Le projet de prison d'Ensisheim en est un exemple. Il est une synthèse des modèles historiques de l'architecture pour exprimer une dramaturgie expressive et morale. Par la polysémie des symboles utilisés, Boudhors a élaboré une harmonisation d'effets variés qui expriment le caractère de ce monument public: la prison avait vocation rédemptrice et moralisatrice. Bien que fortement inspiré par Ledoux, Boudhors n'a pas imité servilement son maître mais il en a fait une réinterprétation savante au service d'une monumentalité expressive et pédagogique.

\section{NOTES}

1. Pour en savoir plus sur les études de l'architecte : UMBRECHT Véronique, "Pierre-Valentin Boudhors (1754-1831), initiateur du néo-classicisme en Alsace» in CHÂTELET Anne-Marie \& STORNE Franck (dir.), Des Beaux-Arts à l'Université. Enseigner l'architecture à Strasbourg. Paris / Strasbourg, Éditions Recherches / École nationale supérieure d'architecture de Strasbourg, vol. 1, 2013, p. 136-143.

2. UMBRECHT Véronique, "Le théâtre de Strasbourg, esquisse d'une longue gestation ", Cahiers alsaciens d'archéologie d'art et d'histoire, $\mathrm{n}^{\circ}$ 54, 2011, p. 107-120.

3. PENY Christine, «Les dépôts de mendicité sous l'Ancien Régime et les débuts de l'assistance publique aux malades mentaux (1764-1790) », Revue d'histoire de la protection sociale, $\mathrm{n}^{\circ} 4,2011$, p. 9-23, [en ligne] https://www.cairn.info/revue-d-histoire-de-la-protection-sociale-2011-1page-9.htm [lien valide en novembre 2021].

4. ABDELA Sophie, La Prison parisienne au XVIII siècle. Formes et réformes, Ceyzérieu, Champ-Vallon, coll. « Époques », 2019.

5. CARLIER Christian, «Histoire des prisons et de l'administration pénitentiaire française de l'Ancien Régime à nos jours ", Criminocorpus, varia, 2009, [en ligne] http:// journals.openedition.org/criminocorpus/246 [lien valide en novembre 2021].

6. ABDELA Sophie, La prison parisienne au XVIII ${ }^{e}$ siècle. Formes et réformes.

7. BLONDEL Jacques François, Cours d'architecture ou traité de la décoration, distribution et construction des bâtiments, Paris, Desaint éditions, tome 2, 1771, p. 455, disponible en ligne https:// gallica.bnf.fr/ark:/12148/bpt6k6558006r.texteImage [lien valide en novembre 2021]. 
8. BENTHAM Jérémy, Panoptique. Mémoire sur un nouveau principe pour construire des maisons d'inspection et nommément des maisons de force. Paris, Imprimerie nationale, 1791, disponible en ligne https://gallica.bnf.fr/ark:/12148/bpt6k114009x.texteImage $[$ lien valide en novembre 2021]. Jeremy Bentham (1748-1832) était un juriste et philosophe anglais qui prônait une justice sociale et œuvra pour modifier les lois pénales. Son panoptique eut une grande influence notamment aux États-Unis.

9. FOUCART Bruno, «Architecture carcérale et architectes fonctionnalistes en France au $\mathrm{XIX}^{\mathrm{e}}$ siècle ", Revue de l'art, $\mathrm{n}^{\circ}$ 32, 1976, p. 38.

10. HARSANY Zoltan-Etienne, «Les prisons de Strasbourg durant la Révolution 1789-1799 ", Annuaire des Amis du Vieux Strasbourg, tome III, 1972-1973, p. 107-132, disponible en ligne https:// gallica.bnf.fr/ark:/12148/bpt6k97527569/f5.item [lien valide en novembre 2021].

11. BATAILLE-WINTERHALTER Gilles \& BATAILLE-WINTERHALTER Béatrice, «Le dépôt de mendicité d'Ensisheim ». Le Généalogiste de Haute-Alsace, n 40, 2004, p. 5-8.

12. Discours préliminaire sur l'établissement en général des prisons, archives départementales du Haut-Rhin, Y 208 à 211, prison centrale d'Ensisheim. Ce texte explicatif avait été envoyé au préfet en appui des plans de la prison. Le texte intégral est à lire dans : UMBRECHT Véronique, « Autour d'une source. Transformer un dépôt de mendicité en prise. Les préoccupations morales et hygiénistes d'un architecte. Discours préliminaire sur l'établissement en général des prisons (1806). », Source(s). Cahiers de l'équipe de recherche Arts, Civilisation et Histoire de l'Europe, n 3, 2013, p. 87-105, disponible en ligne https://arche.unistra.fr/websites/arche/Productions/Publications/ Source_s/Numeros_et_couvertures/Source_s__3_2013.pdf [lien valide en novembre 2021].

13. RABREAU Daniel, La Saline royale d'Arc-et-Senans. Un monument industrie : allégorie des Lumières, Paris, Belin-Herscher, coll. «Les destinées du patrimoine », 2002, p. 43 ; LEDOUX Claude Nicolas, L'architecture considérée sous le rapport de l'art, des mœurs et de la législation, Paris, imprimerie de H.L. Peronneau, tome I, 1804, disponible en ligne https://gallica.bnf.fr/ark:/12148/bpt6k857284.image [lien valide en novembre 2021].

14. Pour en savoir plus, lire: UMBRECHT Véronique, «Pierre Valentin Boudhors (1754-1831), un architecte singulier au service de la ville de Strasbourg ». Cahiers alsaciens d'archéologie d'art et d'histoire, $\mathrm{n}^{\circ}$ 56, 2013, p. 221-235.

15. Discours préliminaire.

16. Située à quelques dizaines de kilomètres d'Ensisheim, la ville de Mulhouse était renommée sous l'Ancien Régime pour ses indiennes de coton.

17. Les trois élévations se trouvent aux archives de la Ville et de l'Eurométropole de Strasbourg.

18. PIRANESI Giovanni Battista, Carceri d'invenzione, 1745-1750. Voir des exemples via http:// www.gizmoweb.org/wp-content/uploads/2014/02/Giovani-Battista-Piranesi.pdf (en italien) [lien valide en novembre 2021].

19. Voir ce modèle architectural dans l'article de RABREAU Daniel, « Le relativisme du renouveau classique dans l'architecture des Lumières ", in GARRIC Jean-Philippe, LEMERLE Frédérique \& PAUWELS Yves (dir.) Architecture et théorie. L'héritage de la Renaissance, actes de colloque organisé par le Centre d'études supérieures de la Renaissance (CESR) de Tours, l'université François Rabelais, l'Institut national d'histoire de l'art et l'École nationale supérieure d'architecture de Paris-Malaquais (Tours le 3 et 4 juin 2009 / Paris le 5 juin 2009), Paris, Institut national de l'histoire de l'art, 2012, disponible en ligne https://books.openedition.org/inha/3422 [lien valide en novembre 2021] ; DE L'ORME Philibert, Architecture, Rouen, 1648. En savoir plus via https:// gallica.bnf.fr/ark:/12148/bpt6k854656s [lien valide en novembre 2021]. 
20. MOSSER Monique, «Le rocher et la colonne: un thème d'iconographie architecturale au XVIII ${ }^{\mathrm{e}}$ siècle ", Revue de l'art, $\mathrm{n}^{\circ} 58,1983$, p. 53-74.

21. MOSSER Monique, "Le rocher et la colonne: un thème d'iconographie architecturale au XVIII ${ }^{\mathrm{e}}$ siècle ", p. 59.

22. RABREAU Daniel, Claude-Nicolas Ledoux (1736-1806), l'architecture et les fastes du temps, Paris, librairie de l'Architecture et de la Ville, 2000.

23. MOSSER Monique, «Le rocher et la colonne : un thème d'iconographie architecturale au XVIII ${ }^{\mathrm{e}}$ siècle ", p. 59.

24. ADHR, Y 211, lettre du 21 février 1807 du ministre de l'Intérieur au préfet du département du Haut-Rhin à Colmar.

25. ADHR, Y 211, lettre du 7 août 1807 de Boudhors au préfet du Haut-Rhin.

\section{RÉSUMÉS}

En 1806, le préfet du Haut-Rhin demande à l'architecte de la ville de Strasbourg Pierre Valentin Boudhors de dessiner les plans de transformation du dépôt de mendicité en prison centrale à Ensisheim. L'architecte refond totalement le bâtiment existant qui devait rassembler les condamnés de plusieurs départements. Formé à Paris notamment dans le cabinet de Chalgrin, il suit le sillon tracé par les architectes de la Révolution pour qui l'architecture était un acte moralisateur. L'édifice participe de l'éducation du citoyen et doit afficher son efficacité éthique : la prison n'a de justification que dans la réinsertion du condamné dans la société. Néanmoins, cette pédagogie se doit de transparaître dans l'aménagement de bâtiments prenant en compte l'hygiène et la moralisation du lieu. L'architecte s'appuie sur son Discours préliminaire pour expliciter son projet. Il développe une "architecture parlante» et y mêle les éléments architecturaux antiquisants, médiévaux et de la Renaissance italienne. Mais l'ensemble de ses dessins puise surtout son inspiration dans la saline d'Arc-et-Senans de Claude Nicolas Ledoux. À partir des bâtiments existants, il trace un axe central qui partage symétriquement les nouveaux bâtiments et organise une succession de cours destinées aux différents types de prisonniers. Il élabore ainsi un complexe architectural qui mêle expression et moralisation.

In 1806, the prefect of the Haut-Rhin asked the architect of the city of Strasbourg Pierre Valentin Boudhors to draw up plans for the transformation of the beggars' home in the central prison in Ensisheim. The architect fully overhauled the existing building which was intended to gather the convicts from several departments. Trained in Paris, especially in the architect's office of Chalgrin, he followed the path of the architects of the Revolution, who were considering architecture as a moralising act. The building participated in the education of the citizen and had to show its ethical efficiency: the prison had no other justification than bringing back the convicts into society. Nevertheless, this pedagogy had to be reflected in the development of buildings taking into account the hygiene and moralisation of the place. The architect explained his project in his Preliminary Speech. He carried out a "self-explaining architecture" ("architecture parlante") while mixing the ancient, medieval and Italian Renaissance architectural elements. Actually, all his drawings were mainly inspired by the Arc-et-Senans saltworks built by Claude Nicolas Ledoux. From the existing buildings, he drew a central axis that symmetrically split the new buildings and organised a succession of yards dedicated to each type of prisoner. Thus, he has designed an architectural complex mixing expression and moralisation. 
INDEX

Keywords : prison architecture, architecture of the first half of the 19th century, functionalist architecture, Ensisheim, Boudhors (Pierre-Valentin), Ledoux (Claude-Nicolas), cave, hygiene, prison

Mots-clés : architecture carcérale, architecture de la première moitié du XIXe siècle, architecture fonctionnaliste, Ensisheim, Boudhors (Pierre-Valentin), Ledoux (Claude-Nicolas), grotte, hygiène, prison

\section{AUTEUR}

\section{VÉRONIQUE UMBRECHT}

Membre associé de l'UR 3400 - ARCHE

Université de Strasbourg

veronique.umbrecht@ac-strasbourg.fr 